\title{
Taxonomy of Aquilegia glandulosa Fischer ex Link and related species (Ranunculaceae) in North Asia
}

\author{
I.V. Kaigalov ${ }^{1}$, A.S. Erst ${ }^{1,2}, N$. A. Tashev $^{3}$, and W. Wang ${ }^{4}$ \\ ${ }^{1}$ Central Siberian Botanical Garden SB RAS, 630090 Novosibirsk, Russia \\ ${ }^{2}$ Tomsk State University, 634050 Tomsk, Russia \\ ${ }^{3}$ University of Forestry, 1756 Sofia, Bulgaria \\ ${ }^{4}$ State Key Laboratory of Systematic and Evolutionary Botany, Institute of Botany, Chinese Academy \\ of Sciences, 100093 Beijing, China
}

\begin{abstract}
The paper presents the annotated checklist and morphological differences of the Aquilegia glandulosa from the nearest related species and provides new data on the distribution of all species from this group. An identification key to the species of Aquilegia glandulosa group from the North Asia is provided and diagnostic characters are discussed.
\end{abstract}

\section{Introduction}

The genus Aquilegia L. (Ranunculaceae) consists of about 110 species and is mainly distributed in the temperate regions of the Northern Hemisphere [9], with 21 species in North America [12], 34 in Asia [4, 9], and 56 in Europe [10]. Aquilegia (known as columbines) possesses unusual floral organs, represented by petaloid sepals, staminodium, and petals with a nectar spur, which allow the study of evolutionary novelties $[8,13]$. The exact number of taxa is still unknown due to spontaneous emergence of new hybrids and stable hybridogenous species [5]. Despite the progress in resolving the systematic relationships of many Aquilegia species using molecular phylogenetic analysis [6], the Asian members are still poorly investigated. Recent morphological and genetic studies [1-3, $5,11]$ revealed greater taxonomic diversity within selected Asian Aquilegia species [4].

\section{Material and methods}

Examination of herbarium material was undertaken in the herbaria LE, MHA, MW, PE, NS, NSK, XJA, XJBI (acronyms follow Thiers (2017+) and Chinese virtual herbaria (http://www.cvh.ac.cn/her). Micromorphology of seed surface was observed and photographed using Scanning Electron Microscope Hitachi S-4700 after sputter coating with gold-palladium.

\section{Results}


Annotated checklist of Aquilegia glandulosa and related species:

Section 1. Glandulosae I.M.Vassiljeva, 1996, Novosti Sist. Vyssh. Rast., 30: 19. - Grex Macranthae Baker, 188, Gard. Chron. Ser. 2, 10: 19, 203.

T y p e: A. glandulosa Fisch. ex Link.

1. A. glandulosa Fisch. ex Link, 1822, Enum. Hort. Berol., 2: 84; A. glandulosa Munz, 1946, Gentes Herbarum, 7: 38; Grubov, 1982, Opred. Sosud. Rast. Mongolii (Key Vasc. Pl. Mongolia): 109, p.p.; Gubanov, 1996, Konsp. Fl. Vneshnei Mongolii (Sosud. Rast.): 48, p.p.; Grabovsk., 2001, Rast. Tsentral. Azii, 12: 128, p.p.; Urgamal et al., 2014, Consp. Vasc. Pl. of Mongolia: 84, p.p. - A. grandiflora Shangin, 1793, Neueste Nord. Beytr. Phys. Geogr. Erd-Völkerbeschreib., 6: 49. - A. alpina var. grandiflora DC., 1818, Syst. Nat., 1: 337. - A. glandulosa var. concolor DC. Prodr., 1: 50. - A. vulgaris var. grandiflora Walp., 1857, Ann. Bot. Syst., 4: 25. - A. vulgaris subsp. glandulosa Brühl, 1893, Journ. Asiatic Soc. Bengal, 15(2): 61. - A. vulgaris subsp. glandulosa var. vera Brühl, op. cit. 303. - A. brevicalcarata Kolokoln. ex Serg., 1956, Sist. Zametki Mater. Gerb. Krylova Tomsk. Gosud. Univ. 79: 6.

T y p e: «Hab. in Sibiria» (LE!).

Habitat: On alpine and subalpine meadows, nival lawns, along the banks of mountain streams, on talus, rocks.

Distribution: Mongolia (Bayan-Ulgiy, Bayankhongor, Bulgan, Gov-Altai, Dornod, Selenge, Tuve, Uvs, Khovd, Khentei), Russia (Altai Territory, Krasnoyarsk Territory, Transbaikal Territory, Irkutsk Region, Republic of Altai , Republic of Buryatia, Republic of Tyva, Republic of Khakassia, Republic of Yakutia), China (Xinjiang), Kazakhstan (East Kazakhstan region).

2. A. jucunda Fisch. et Lallem., 1839, Index sem. hort. petrop., 6: 43; Ledeb., 1843, Fl. Ross., 1: 736. - A. glandulosa var. discolor DC., 1824, Prodr., 1: 50. - A. glandulosa var. jucunda (Fisch. et Lallem.) Baker, 1878, Gard. Chron. n.s., 10: 203. - A. glandulosa non Fisch. ex Link, auct.: Grubov, 1982, Opred. Sosud. Rast. Mongolii (Key Vasc. Pl. Mongolia): 109, p.p.; Gubanov, 1996, Konsp. Fl. Vneshnei Mongolii (Sosud. Rast.); Grabovsk., 2001, Rast. Tsentral. Azii, 12: 128, p.p.; Urgamal et al., 2014, Consp. Vasc. Pl. of Mongolia: 84, p.p.

T y p e: «Hab. una cum praecedente in Sibiriae montibus» (LE!).

Habitat: on rocky outcrops, talus, along the edges of forests, among bushes, in larch forests.

Distribution: Russia (Altai Krai, Krasnoyarsk Krai, Transbaicalia Krai, Irkutsk region, Altai Republic, Buryatia, Tyva, Khakassia, Yakutia), China (Xinjiang), Kazakhstan (Almaty and East Kazakhstan Oblast), Mongolia (Bayan-Ulgiy, Gov-Altai, Khovd, Khuvsgel).

3. A. daingolica A. Erst et Schaulo, 2013, Syst. notes, 108: 15. - A. $\times$ gubanovii Kamelin, Byull. Moskovsk. Obshch. Isp. Prir., Otd. Biol., 96(6): 114, nom. nud.

T y p e: North-western Mongolia, the lake Daingol, western slopes. July 27-29, 1909 V.V. Sapozhnikov (Holotype TK!); p a r a t y p e: Western Mongolia, Altai, the lake Daingol, steppe slopes. July 5, 1906. V.V. Sapozhnikov (TK!).

Habitat: On alpine meadows, gravelly placers, among stones, near snowfields.

Distribution: Mongolia. Bayan-Ölgii (endemic).

4. A. xinjiangensis Erst, 2017, Phytotaxa, 316 (2): 122.

T y p e: China. Xinjiang: [Altay Prefecture], Qinghe [Qinggil] County, upstream of Daqing River, $2100 \mathrm{~m}$, subalpine meadows or wetland, 7 July 2002, B. Wang 02-536 (holotype, XJA-0003378!); p a r a t y p e: China. Xinjiang: Altay Prefecture, Fuhai (Burultokay) County, Fuhai Forest Farm, mountainous coniferous forest, 4 August 1964, G.L. Zhu et al. 6059 (PE00105591!).

Habitat: Subalpine meadows and mountainous coniferous forests, 2000-2500 m a.s.l. 
Distribution: China. Xinjiang: Altay Prefecture (endemic).

Identification key to the Aquilegia glandulosa and related species (Ranunculaceae) in North Asia:

Flowers concoloured (light blue, blue, purple, white, cream), spurs $0.6-1 \mathrm{~cm}$ long, lamina ovate, spreading, lamina apex bent inwards, follicles 8-15

- Flowers 2-coloured (sepals and spurs blue, petal laminae white or yellowish), spurs 1$3 \mathrm{~cm}$ long, lamina spatulate or oblong, suberect or parallel to floral axis, lamina apex bent

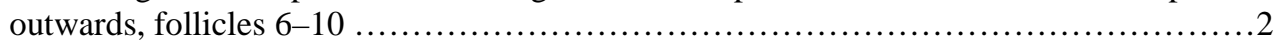

2. Anthers yellow, spurs convergent, spur tip hooked, lamina spatulate, follicle covered with glandular hairs ................................................... A. jucunda

- Anthers blackish, spurs divergent spur tip straight or clightly curved, lamina oblong,

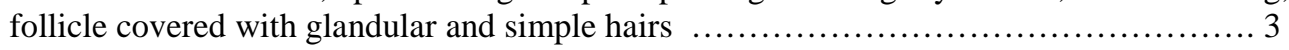

3. Sepals sreading, spurs tip straight, base of spurs inflated, lamina $1.2-1.8 \mathrm{~cm}$ long A. daingolica

- Sepals perpendicular to floral axis, spurs tip slightly curved, base of spurs not inflated (thin), lamina $2-3.5 \mathrm{~cm}$ long A. xinjiangensis

\section{Discussion}

Aquilegia xinjiangensis [Fig. 1]is morphologically similar to A. daingolica [Fig. 2] from NW Mongolia in having 2-coloured petals and dark anthers. However, it differs by the thin spurs, which are slightly shorter than the laminae and slightly curved at the top [4]. Aquilegia daingolica - stabilized species of hybrid genetic origin, in whose formation $A$. glandulosa, A. oxysepala and probably A. sibirica took part. It is similar to A. glandulosa [Fig. 3] in the size of flowers and form of fruit aggregates. The species is close to hybrid genetic A. oxysepala in dark colored anthers and black clublike ends of spurs. Spurs' features distinguish Aquilegia daingolica from all Asian representatives of the genus. Aquilegia daingolica has basally inflated, almost straight spurs longer than or equal in length to the laminae [1]. All species of this group (A. daingolica, A. xinjiangensis, A. jucunda, A. glandulosa s.str.) are distinguished by the tuberculate seed surface. Aquilegia ochotensis Voroshilov (1981: 104) and A. transsilvanica Schur cannot be assigned to this group, as was proposed by Voroshilov (1981) and Vassiljeva (1996). Aquilegia ochotensis, a species occurring in Far Eastern Russia, differs from other species of this group by the striate seed surface and abaxially pilose leaf blades. Aquilegia transsilvanica is nested within phylogenetic 'Group VI' that unites European species [6]. Such placement is clearly supported by morphological data [7], including the striate seed surface.

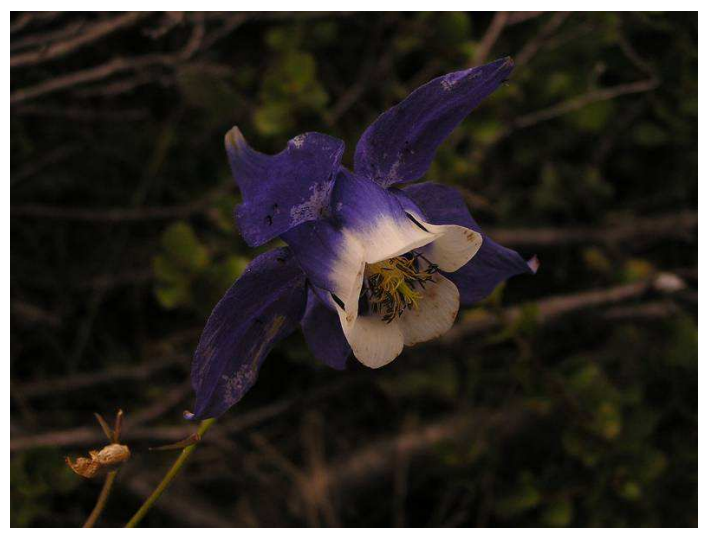

Fig. 1. Aquilegia xinjangensis Erst 


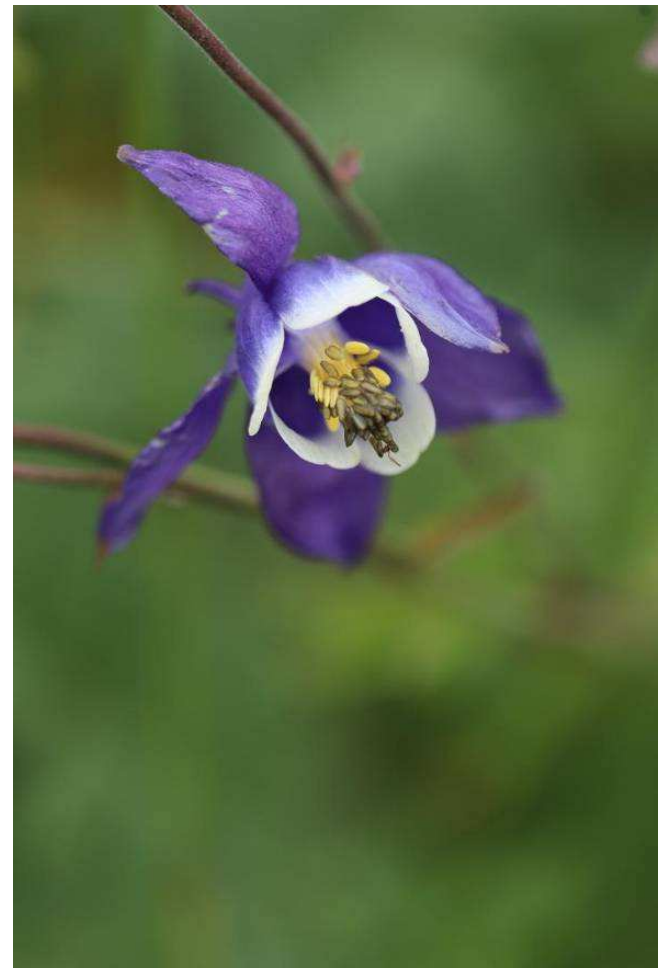

Fig. 2. Aquilegia daingolica A. Erst et Schaulo

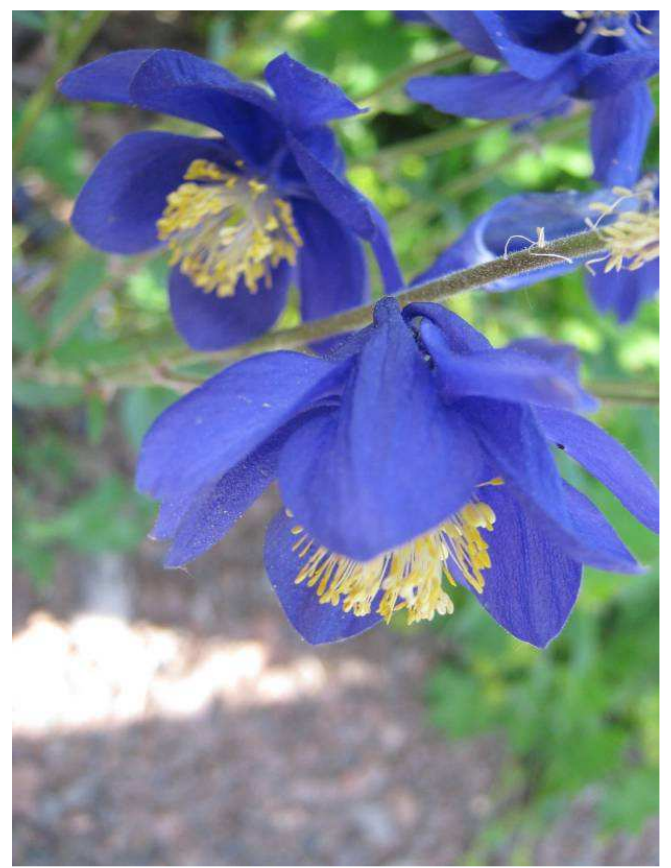

Fig. 3. Aquilegia glandulosa Fischer ex Link 


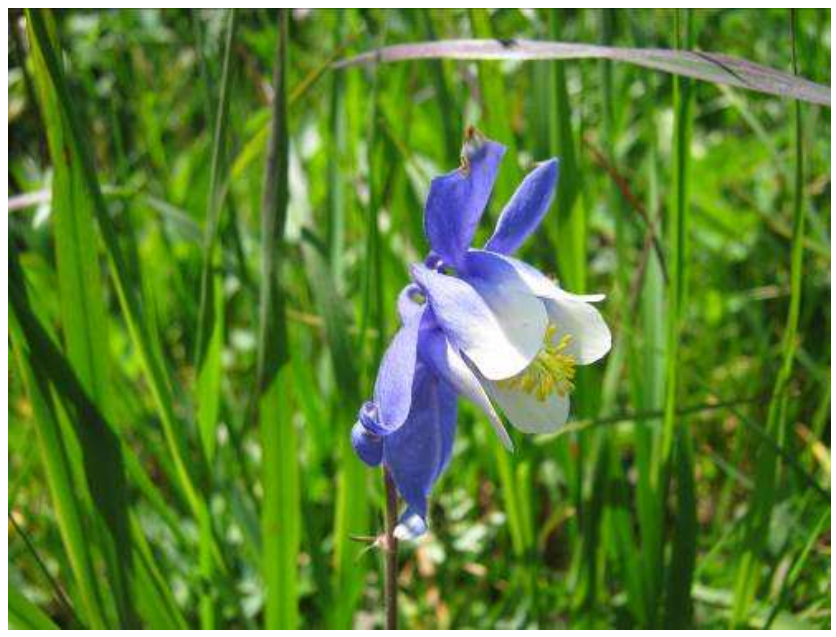

Fig. 4. Aquilegia jucunda Fisch. et Lallem.

Acknowledgements. We are grateful to the curators and staff of several herbaria for making the specimens available for our study. This research was supported by the Russian Science Foundation, Project \#19-74-10082.

\section{References}

1. A.S. Erst, D.N. Shaulo, A.A. Kuznetsov, Sist. Zam., 108 (2013)

2. A.S. Erst, D.N. Shaulo, A.I. Schmakov, Turczaninowia, 16 (3) (2013)

3. A.S. Erst, A.P. Sukhorukov, Acta Botanica Gallica, 162 (3) (2015)

4. A. Erst, W. Wang, Sh. Yu, K. Xiang, J. Wang, D. Shaulo, S. Smirnov, M. Kushunina, A. Sukhorukov, M. Nobis, Phytotaxa, 316 (2) (2017)

5. A. Erst, O. Vaulin, Rus. J. of Genetics, 4 (1) (2014)

6. S. Fior, M. Li, B. Oxelman, R. Viola, S. Hodges, L. Ometto, C. Varotto, New Phytologist, 198 (2013)

7. Y. Kobiv, Ukr. Bot. J., 69 (4) (2012)

8. E.M. Kramer, L. Holappa, B. Gould, M.A. Jaramillo, D. Setnikov, P. Santiago, Plant Cell, 19 (2007)

9. Y. Luo, A.S. Erst, C.-X. Yang, J.-P. Deng, L. Li, Phytotaxa, 348 (4) (2018)

10. E. Nardi, Il genere Aquilegia L. (Ranunculaceae) in Italia. (Edizione Polistampa, 2016)

11. D. N. Shaulo, A. S. Erst, Turczaninowia, 13 (3) (2010)

12. A. T. Whittemore, Rodriguesia, 66(4) (1997)

13. C. Voelckel, J. O. Borevitz, E. M. Kramer, S. A. Hodges, Plos One, 5(3) (2010) 